\title{
Resistance of a steel-concrete hybrid thermal break system to low cycle fatigue under thermal actions
}

\author{
B. Le Gac ${ }^{a, b *}$, P. Keo ${ }^{a}$, H. Somja ${ }^{a}$ and F. Palas ${ }^{b}$ \\ ${ }^{a}$ National institute of applied sciences, Department of Civil Engineering, Rennes, France \\ ${ }^{\mathrm{b}}$ INGENOVA, Civil Engineering Office, Rennes, France \\ *corresponding author, e-mail address: benoit.le-gac@insa-rennes.fr
}

\begin{abstract}
External insulation is the most widely used technique in Northern and Continental Europe. This technique generates thermal bridges where the building facade has some projecting element like balconies. The thermal requirements of actual standards lead to restore the continuity of the insulation at the interfaces by using thermal break systems (TBS). They are usually made of a box containing the insulation material, and a minimalist structural system able to transmit the shear force and the bending moment from the balcony to the wall. In most cases, structural elements are made of stainless steel, as it is less heatconducting than normal steel. The paper focuses on a specific TBS, that uses shear keys and steel profiles to ensure the transfer of forces.

TBS are also submitted to important horizontal cyclic shear deformations, provoked by the variations of the dimensions of the balconies due to climatic effects. The objective of the study presented in the paper is to show that significant yielding under these cyclic actions can be accepted during service life. First experimental cyclic loading tests have been performed in order to characterize the behaviour of the TBS, as well as its fatigue strength. Then the loading has been defined on the basis of the database of the ECA\&D, the European Climate Assessment and Dataset. Finally, the fatigue resistance of the system has been verified. It is shown that the developed TBS can resist to fatigue loading for large lengths of balconies, while exhibiting significant yielding during service life.
\end{abstract}

Keywords: Hybrid concrete-steel, Thermal break, low cycle fatigue, thermal actions.

\section{Introduction}

The level of energy-performance requirements in buildings has substantially increased over the last twenty years. As the thickness and the efficiency of the insulation of the walls increase, the energy lost in the building is now mostly due to the discontinuity of the insulation, where so called thermal bridges are created. These thermal bridges induce moreover a local condensation of water that can cause a deterioration of the internal coating of the building and even a degradation of the indoor air quality due to the development of decay. In the specific case of buildings with an external insulation, thermal bridges develop at locations where the building facade has projecting element such as balconies, see Fig. 1.

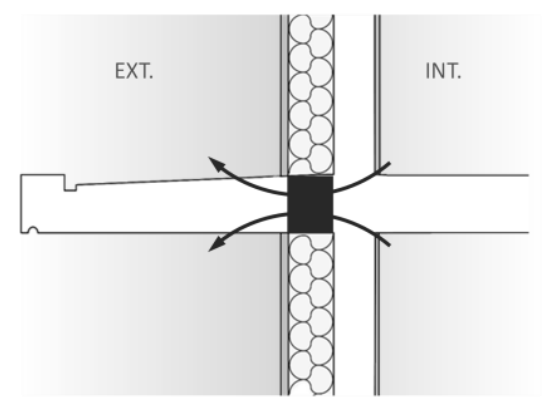

Fig. 1. Balcony thermal bridge and TBS location.

As a consequence, thermal bridges must be reduced by the use of appropriate solutions like thermal break systems (TBS). Usual TBS are made of a box containing the insulation material, and a minimalist structural system able to transmit the shear force and the bending moment from the balcony to the wall. In most cases, 
structural elements are made of stainless steel, as it is less heat-conducting than normal steel.

The structural role of the TBS is not only to resist to vertical forces, wind or even seismic action, but also to absorb the relative displacements induced by the thermal expansion of the balcony. This critical point is discussed in the specific case of a TBS called SUNE. It is an assembly of different components consisting of tensile rebars, U-shaped steel sections, and special shear keys, see Fig. 2 .

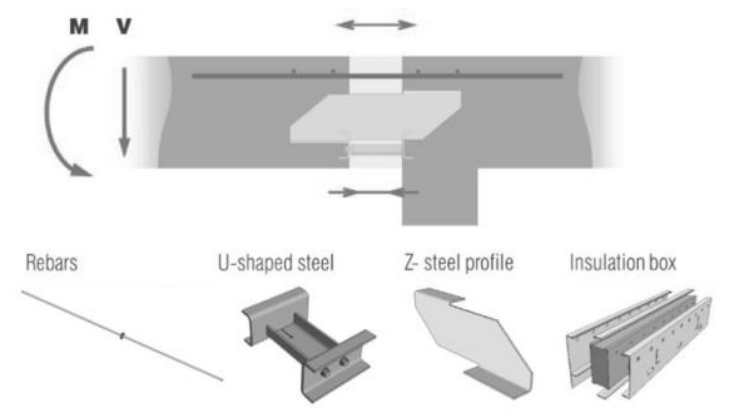

Fig. 2. SUNE thermal break system.

The tensile rebars and the U-shaped steel sections are used to balance the tension and compression forces due to bending while the shear key is used to resist the vertical shear force. The web of the U member presents longitudinal slots at each end in order to provide some horizontal flexibility. On the lintel side, it relies on an end plate with a U section, with flanges embedded in the concrete. On the balcony side, a rectangular end plate is welded on the $U$ member. This end plate is connected by two screws to a U-shaped end plate embedded in the concrete in the same way as on the other side.

Mechanical performances of the SUNE under vertical loads are presented in [1]. To contribute to the thermal performance of the TBS, duplex stainless steels with a limit of elasticity greater than $600 \mathrm{MPa}$ and $550 \mathrm{MPa}$ are used for the rebars and the steel profiles, respectively. A mineral wool is used as insulation material in the insulation box of $100 \mathrm{~mm}$ thick. The use of those materials leads to a good thermal performance with linear thermal transmittance values below $0.27 \mathrm{~W} /(\mathrm{m} \mathrm{K})$.

Being located outside the building thermal envelope, the balcony suffers climatic hazards and is caused to expand or shorten following climatic conditions (outside temperature, solar radiation, etc.). The thermal break is placed in line with the insulation and thus ensures the connection between the outside balcony and the inner floor slab. The latter is located inside the building envelope; consequently it only undergoes low changes in temperature. The thermal break is therefore subjected to shearing induced by the horizontal deformation of the balcony as a function of the outside temperature variations. For that reason, the components of TBS must be designed to be able to sustain such deformations. The bars and Z-profile have sufficient horizontal flexibility to deform freely under the thermal forces, but the U-shaped steel element undergoes yielding even under frequent actions.

The objective of the work presented in this paper is to prove that this yielding is admissible and does not reduce the capabilities of the TBS during its service life. Accepting yielding at SLS requires a verification against low-cycle fatigue, given the fact that the yielding may occur several times during the service life of the element.

In this paper, the verification of the thermal break system SUNE against low-cycle fatigue loads is performed. To do so, cyclic loading tests of the TBS are conducted. Their results serve to establish the cyclic force-displacement relationship, as well as the fatigue design curve of the system. The fatigue design curve is then used to verify the fatigue strength of the thermal break system under the deformations of the balcony generated by the temperature variation outside the building. The latter is originally obtained from a European database. Finally, the verification of TBS against thermal loading is done by determining the damage accumulation developed during the building life. Several meteorological stations as well as balcony lengths are considered in the final parametric study.

\section{Low cycle fatigue tests}

The mechanical behavior of the TBS under cyclic horizontal loads is evaluated through lowcycle fatigue tests. The specimen consists of the balcony, the negative bending moment zone of the adjacent RC slab, and the balcony-slab connection component (TBS), see Fig. 3. Concrete class is $\mathrm{C} 25 / 30$. To consider the gravity load at service limit state for frequent load combination, a block of concrete is suspended on the balcony. The cyclic horizontal action is provided by a hydraulic jack, see Fig. 4 . 


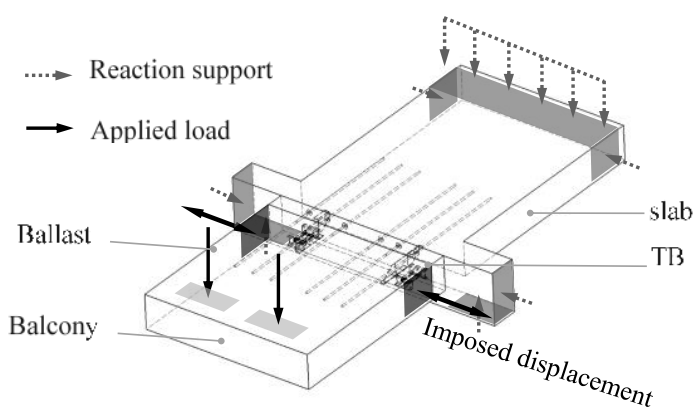

Fig. 3. Actions on the test specimen.

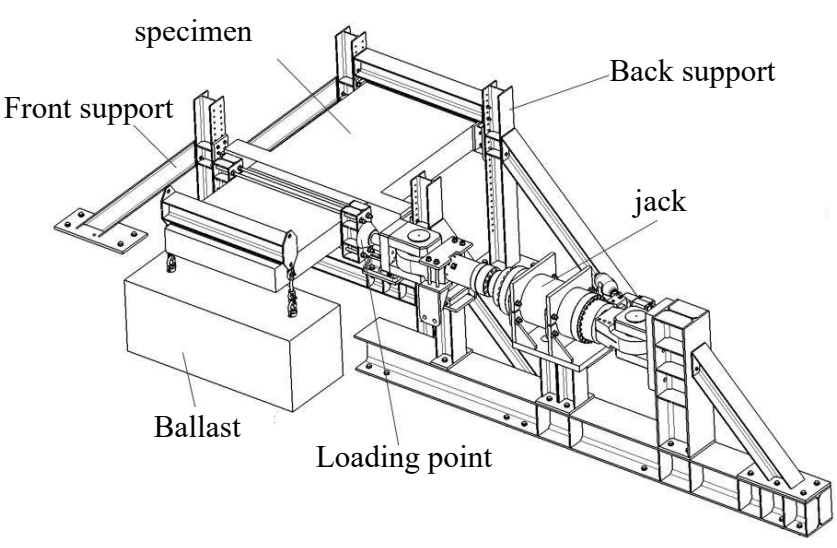

Fig. 4. Fatigue test set-up.

Eight specimens are considered in the experimental test program as required by $\mathrm{NF}$ A03-403 [2] to produce the resistance fatigue curve, which covers at least 4 points with different numbers of cycles.

The loading procedure is divided in two phases. First, a series of cycles with an increasing amplitude is applied in order to get information on the load-displacement curve of the specimen, see Fig. 4. At each level, three cycles are made. Then the amplitude of the loading is maintained (see Table 1) and cycles are applied up to the collapse of the specimen.

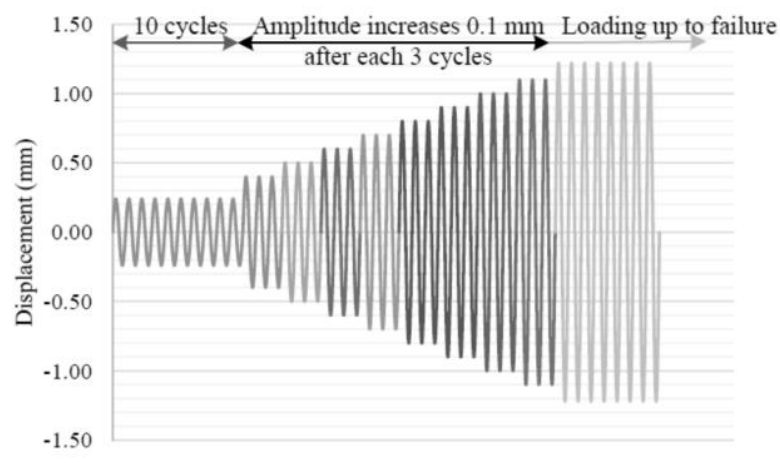

Fig. 5. Example of loading procedure.
Table 1. Testing loading amplitudes (mm)

\begin{tabular}{cccccc}
\hline CH1/2 & CH3 & CH4 & CH5/6 & CH7 & CH8 \\
\hline 1.22 & 2.44 & 1.83 & 1.52 & 2.75 & 5 \\
\hline
\end{tabular}

The force-displacement curves under increasing amplitude show a hardening plateau provoked by the yielding of the stainless steel, as well as a pinching due to the concrete damaging, see Fig. 6. As a consequence, a coupled damageplasticity model is developed to represent the behavior of the TBS.

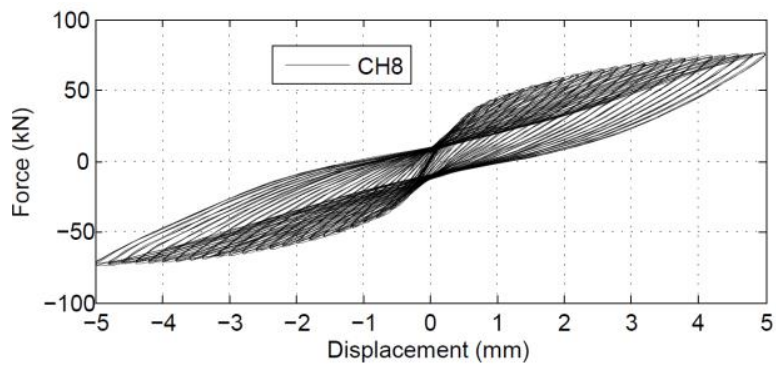

Fig. 6. Force-displacement behavior - $\mathrm{CH} 8$ test

In the constant amplitude phase, Cracks appear in the U-shaped steel member, first starting from the end of the slot, then also in the weldings of the end plates, see Fig. 7. For larger amplitudes, the collapse is attained by crushing of concrete. The specimen is considered as collapsed when a rapid increase of the rotation of the TBS is observed.

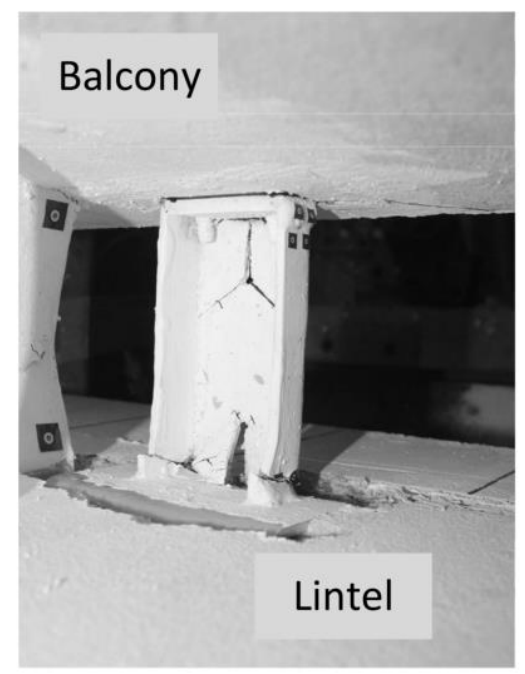

Fig. 7. Typical damaging of a U-shaped profile

The evolution of the rotation with the number of half-sine cycles is presented Fig. 8, and the force and rotation at collapse $F_{u}$ and $\theta_{u}$ in Table 2 , as well as the number of cycles at failure $N_{f}$. $N_{f}$ is expressed in half cycles in all the paper. 


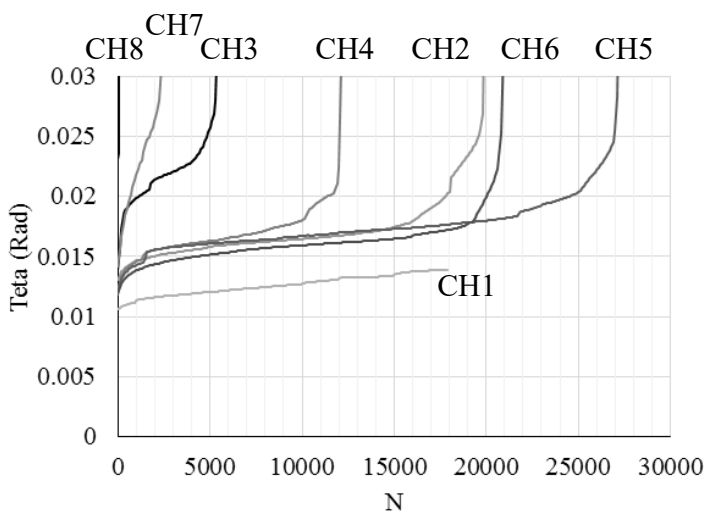

Fig. 8. Evolution of the rotation

Table 2. Testing loading amplitudes (mm)

\begin{tabular}{cccc}
\hline Specimen & $\begin{array}{c}\boldsymbol{\theta}_{\boldsymbol{u}} \\
(\mathbf{r a d})\end{array}$ & $\begin{array}{c}\boldsymbol{F}_{\boldsymbol{u}} \\
(\mathbf{k N})\end{array}$ & $\boldsymbol{N}_{\boldsymbol{f}}$ \\
\hline $\mathrm{CH} 1$ & 0.0139 & 37.8 & 36000 \\
$\mathrm{CH} 2$ & 0.0174 & 35.0 & 30000 \\
$\mathrm{CH} 3$ & 0.0229 & 47.0 & 8000 \\
$\mathrm{CH} 4$ & 0.0180 & 40.3 & 20000 \\
$\mathrm{CH} 5$ & 0.0255 & 38.0 & 54000 \\
$\mathrm{CH} 6$ & 0.0172 & 37.9 & 36000 \\
$\mathrm{CH} 7$ & 0.0295 & 47.3 & 4600 \\
$\mathrm{CH} 8$ & 0.0388 & 67.1 & 68 \\
\hline
\end{tabular}

\section{Low cycle design criterion}

To perform fatigue analysis, two common empirical approaches can be used : stress-life or strain-life method. In the stress-life approach, the life of a structural component is estimated based upon the magnitude of the alternating stresses. One of the major drawbacks of this approach is that it ignores the actual material response and treats all the behavior as elastic. This generally results in an overestimation of the fatigue life when the plastic strain contribution is significant, particularly for the stainless steel. Thus, the stress-life method is not applicable when the plastic strain is not negligible, in lowcycle fatigue for example. Alternatively, the strain- life approach estimates fatigue life using the total strain amplitude, including both the plastic and elastic strain contributions. Thus, it can be applied in low-cycle fatigue where significant plastic strains are present.

For metallic materials, the Manson-Coffin strain life equation $[3,4]$ is able to give a prediction of the fatigue life for a cyclic loading with constant strain amplitude $\varepsilon_{\mathrm{a}}$ and zero mean stress in both elastic and plastic regimes :

$\varepsilon_{a}=\varepsilon_{a}^{e}+\varepsilon_{a}^{p}=\frac{\sigma_{f}}{E}\left(N_{f}\right)^{b}+\varepsilon_{f}^{\prime}\left(N_{f}\right)^{c}$
Where $\varepsilon_{a}^{e}$ is the elastic strain, $\varepsilon_{a}^{p}$ the plastic strain, $\sigma_{f}^{\prime}$ the stress amplitude, $N_{f}$ the fatigue life, and $b$ and $c$ constants determined experimentally.

The mean stress influences very significantly the total fatigue life [5]. In conjunction with the local strain life approach, many models have been proposed to quantify the effect of mean stresses on fatigue behavior. One of the commonly used models is the so-called SWT model, that modifies the Manson-Coffin equation by taking into account the maximum tensile stress $\sigma_{\max }$.

$\sigma_{\max } \varepsilon_{a}=\frac{\left(\sigma_{f}\right)^{2}}{E}\left(N_{f}\right)^{2 b}+{\sigma_{f}^{\prime}}^{\prime} \varepsilon_{f}^{\prime}\left(N_{f}\right)^{b+c}$

Similar to SWT model, an energy-based fatigue criterion was proposed by Jahed and Varvani-Farahani [6] to assess the fatigue lives of engineering components, expressed as :

$\Delta E_{f}=E^{\prime}{ }_{e}\left(N_{f}\right)^{B}+E_{f}^{\prime}\left(N_{f}\right)^{C}$

Where $\Delta E_{f}$ is the energy due to the applied loading, $E_{f}^{\prime}$ the fatigue toughness, $E^{\prime}{ }_{e}^{\text {the }}$ strength coefficient, $C$ the toughness exponent and $B$ the fatigue strength exponent. In this study, this equation has been simplified by neglecting the elastic energy :

$\Delta E_{f}^{\max }=F_{\max } x_{a}=E_{f}^{\prime}\left(N_{f}\right)^{C}$

Where $F_{\max }$ is the maximum force during loading cycle, and $x_{a}$ is the displacement amplitude.

Hence, the energy-fatigue life curve for TBS is constructed based on Eq. (4), see Fig. 15. The mean model parameters are obtained by applying a linear regression analysis, and the characteristic and design curve are obtained following the method proposed in annex D of EN1990 [7].

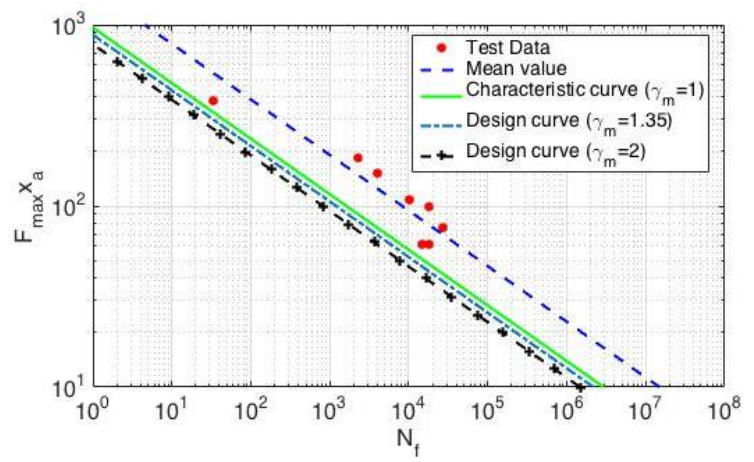

Fig. 9. $\Delta E_{f \max }-N_{f}$ curves 
The choice of partial factor $\gamma_{m}$ value can be taken based on the failure modes of the specimens. It has been observed that the failure modes of the specimen subjected to fatigue loading are mostly related to the fracture of the steel components. As a consequence, it may seem reasonable to adopt $\gamma_{m}=1.35$ as proposed in EC3-1-9 [8] for non-fail-safe structural details poorly accessible. However, this value is defined for polycyclic fatigue while low-cycle fatigue is here under consideration. As a consequence, three different values of $\gamma_{m}$ will be considered to get some information on the sensibility of the design of the TBS to $\gamma_{m}$ : $\gamma_{m}=1,1.35,2$.

\section{Thermal action}

As mentioned in Section 1, the balcony suffers climatic hazards and is caused to expand or shorten following the variation of the temperature outside the building. This variation is computed based on the European Climate Assessment \& Dataset (ECA\&D) [9]. ECA\&D is receiving data from 68 participants for 63 countries and the ECA dataset contains 43271 series of observations for 12 elements at 10586 meteorological stations throughout Europe and the Mediterranean zone.

Five different stations have been chosen in France for this study in order to see what differences can exist between a large city (Paris), places submitted to an oceanic climate (Rennes and Bordeaux), a continental one (Strasbourg) and a mountainous one (Embrun). Recordings cover a period of about 100 years, varying from station to station.

The comparison of maximum and minimum shade air temperature distribution with the one given by the default values of EN 1991-1-5 [10] is made Fig. 10 and 11. As could be expected, some differences exist. It has been decided to scale the temperature signals of ECA\&D data base to fit the $5 \%$ fractile of extreme temperatures of the national annex of the Eurocode, in order to comply with the level of safety imposed by this actual standard, as shown on Fig. 12 and 13.

The effect of solar radiation on extreme values has been added, and then the horizontal deformation $x_{a}$ has been determined in function of the balcony length $L_{b}$ and the coefficient of thermal dilatation $\alpha=10^{-5}$ :

$x_{a}(t)=\alpha \Delta T(t) L_{b} / 2$

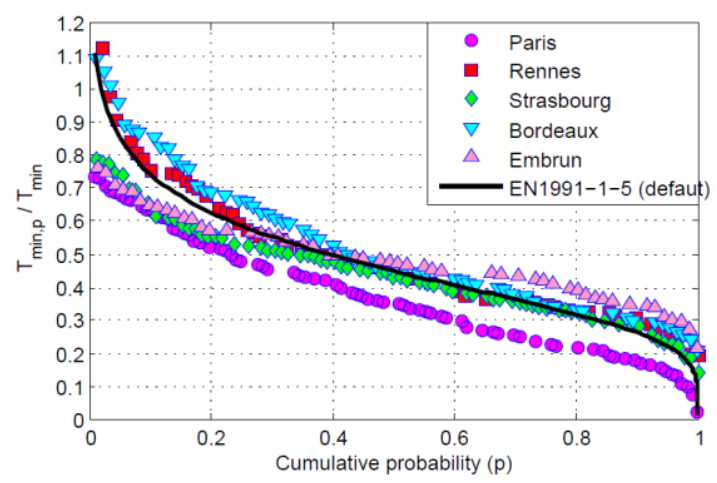

Fig. 10. Distribution of minimum temperatures

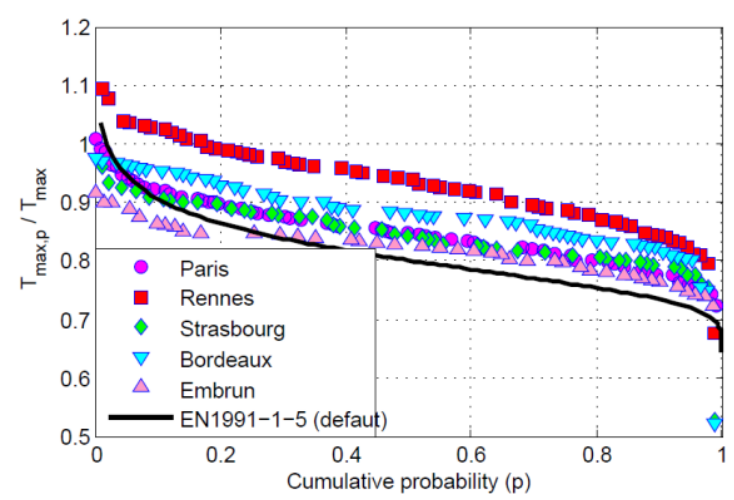

Fig. 11. Distribution of maximum temperatures

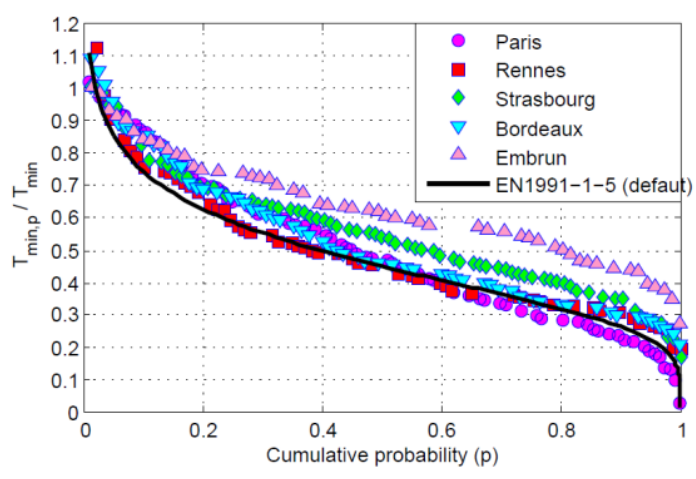

Fig. 12. Adapted ECAD minimum temperatures

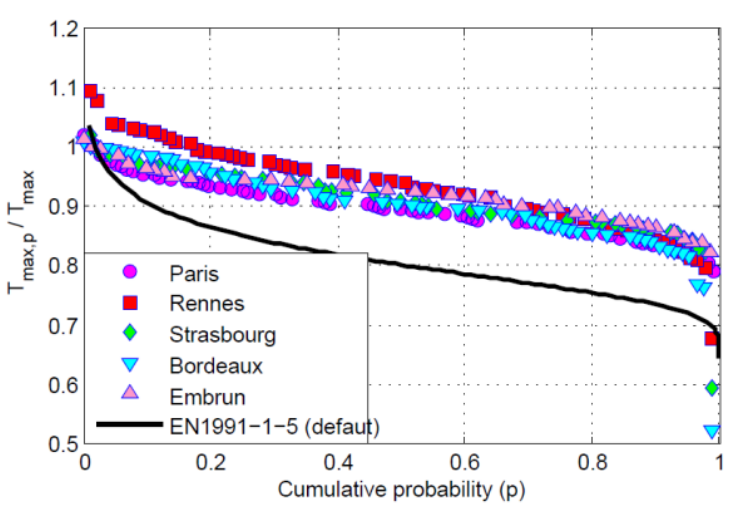

Fig. 13. Adapted ECAD maximum temperatures 


\section{Verification to thermal loading}

The verification of TBS against thermal loading can be performed by determining the damage accumulation developed during the building life. This can be done for an existing building where the distribution of temperature is already known. To design a new building where the climate surrounding the building is not yet known during the building life, a probabilistic approach has to be developed.

It is assumed that the statistical distribution of the climatic action can be computed on the basis of the distribution of the recordings of ECA \& $\mathrm{D}$, transformed as explained in previous paragraph in order to get thermal amplitudes compliant with actual European standards. The climatic action in fatigue is defined on the basis of a duration of one year. The annual accumulated damage $D_{y}$ of the year $i$ is then obtained from :

$D_{y, i}=\sum_{j=1}^{m} \frac{n_{E, j}}{N_{f, j}}$

where $m$ is the number of different displacement amplitudes $x_{a, j}$ obtained from rainflow counting technique [11]; $n_{E, j}$ is the number of semi-sinusoidal cycles of each rainflow displacement amplitude $x_{a, j}$; and $N_{f, j}$ is the number of semi-sinusoidal cycles to failure obtained from $\Delta E_{\max }-N_{f}$ design curve for each rainflow displacement amplitude $x_{a, j}$. In order to use $\Delta E_{\max }-N_{f}$ design curve, the maximum absolute-generalized forces during loading cycle, $F_{\max }$, is required. The analysis with the annual displacement signal is then primarily performed to capture the corresponding force signal acting on the structure by using the coupled damage-plasticity model.

Processing all the data of ECAD for one station gives a set of values of $D_{y, i}$ that is used to obtain an estimation of the mean value denoted $\widehat{D D}_{y}$ and of the standard deviation, $\sigma \widehat{D}_{y}$.

The service life is fixed to 50 years in Eurocodes for usual buildings, and the related accumulated damage denoted $D_{50}$ has to be limited to $1 . D_{50}$ is a random variable equal to the sum of 50 annual damages. Each annual damage is supposed to be independent. Hence, according to the central limit theorem, the data set of $D_{50}$, which is a sum of independent random variables, follows a normal distribution $N\left(50 \widehat{\mu D}_{y}\right.$, $50 \sigma \widehat{D}_{y}^{2}$ ) even if $D_{y, i}$ is not normally distributed.
Within a data sample size equal to $n_{\text {year }}$, the characteristic value of $D_{50, k}$ can be obtained using the following expression :

$D_{50, k}=50 \mu \widehat{D}_{y}+t_{v} \sqrt{50} \widehat{\sigma D_{y}}$

It is worth mentioning that the available data signal from ECA\&D is usually greater than 50 years and that the value of $t_{v}$ is determined by using the inverse value of Student's cumulative distribution function, multiplied by $\sqrt{1+1 / n_{\text {year }}}$. Since the $\Delta E_{\max }-N_{f}$ design curve is used to determine the damage parameter, the characteristic value $D_{50, k}$ is the design value $D_{50, d}$.

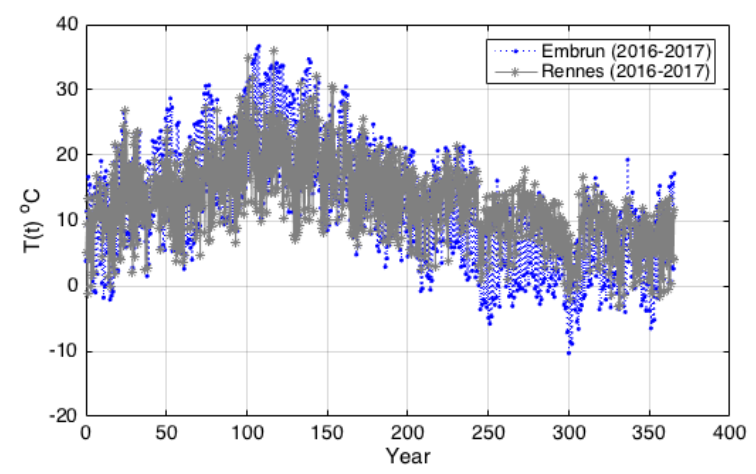

Fig. 14. Factorized Temperature signal in Embrun and Rennes over one year

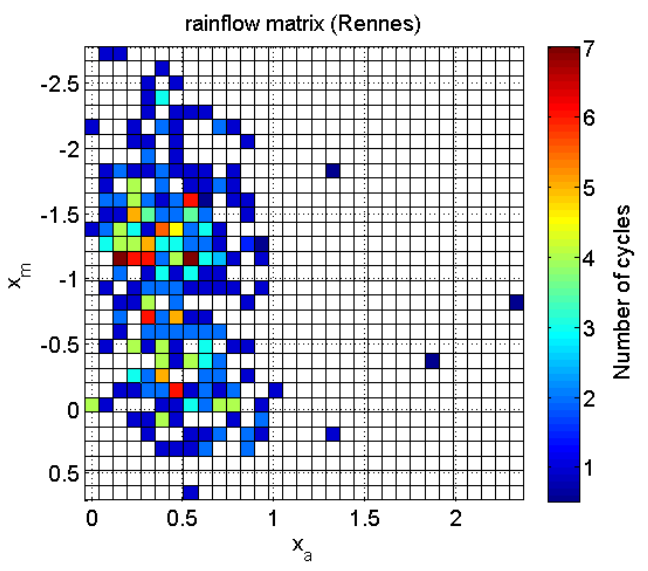

Fig. 15. Rainflow matrix for Rennes

As an illustration, detailed results are presented for the cities of Embrun and Rennes. First the factorized temperature signal is presented in Fig. 14 over one year. It is clear that variations are larger in Embrun, located in the Alps, than in Rennes, located near the Atlantic Ocean. The result of the rainflow counting algorithm is presented in Figs. 15 and 16 for a balcony with a length $L_{b}=19 \mathrm{~m}$, where $x_{m}$ is the mean value of the displacement of the half-cycle, and $x_{a}$ the amplitude. There are only few data 
with large amplitudes, corresponding to the annual variations of temperature.

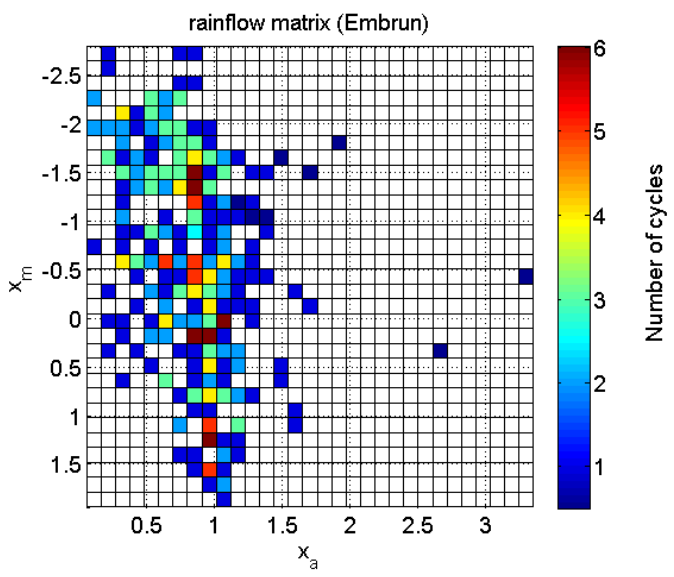

Fig. 16. Rainflow matrix for Embrun

Fig. 17 shows the evolution of the annual accumulated damage, still for $L_{b}=19 \mathrm{~m}$. It can be seen that the evolution of $D_{y}$ is very random. $D_{y}$ during the last 50 years at Rennes city, featuring an oceanic climate, is quasi constant compared to the one in Embrun, in the Alps, which is very fluctuating with a growing global trend. On the contrary, the damaging in Paris is rather decreasing. Bordeaux and Strasbourg give intermediate levels of damaging.

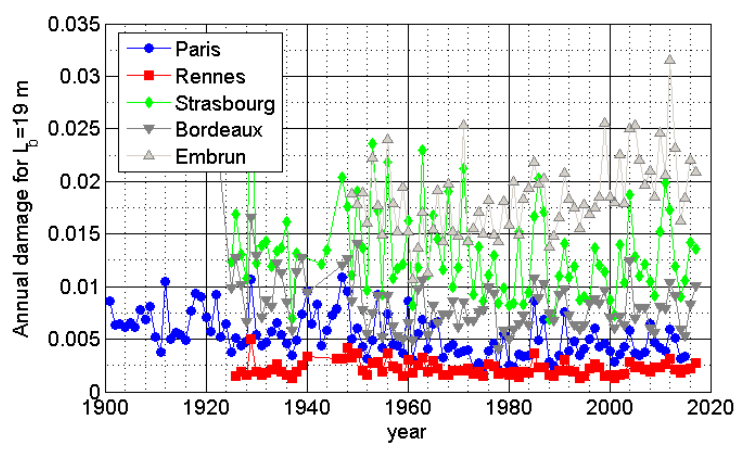

Fig. 17. Evolution of $D_{y}$ for $L_{b}=19 \mathrm{~m}$

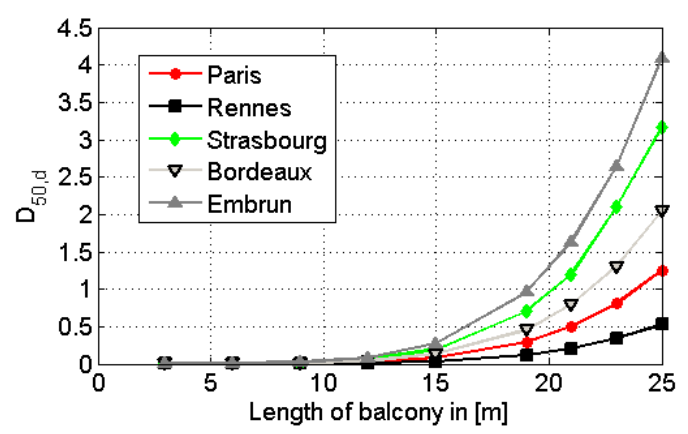

Fig. 18. Evolution of $D_{50, d}$ for $L_{b}=19 \mathrm{~m}, \gamma_{m}=1$

The evolution of the 50-year building life damage $D_{50, d}$ is calculated for the five different cities under consideration for a safety coefficient $\gamma_{m}$ equal to 1. Its evolution in function of the balcony length is illustrated in Fig. 18.

As could be expected, the life-time accumulated damage is larger in Embrun. Strasbourg, which is located in the east of France and is subject to a continental climate, gives also large damaging, while Rennes gives the lower ones. Bordeaux and Paris give intermediate values. These differences are consistent with the climatic zones defined in the Eurocodes.

The evolution of the damaging with various values of the safety coefficient $\gamma_{m}$ is computed for different lengths of balconies in the most unfavorable city, i.e. Embrun, as illustrated in Fig. 19. The TBS can sustain fatigue load during the building service life for a balcony length up to $16.5 \mathrm{~m}, 18 \mathrm{~m}$ and $19 \mathrm{~m}\left(D_{50, d}=1\right)$ for a safety factor $\gamma_{m}=2,1.35$ and 1 , respectively. It appears thus that the safety factor does not substantially influence the possible balcony length. For the fact that the balcony is, in general, made with a length smaller than $12 \mathrm{~m}$, the use of $\gamma_{m}=2$ already gives a large margin. Such large margin provides a proof that it is possible to develop stresses in a plastic range in SLS without the risk of being collapsed during the building service life. However, a reliability analysis in the safety format with larger database of the cities is needed to determine the actual value of the safety factor.

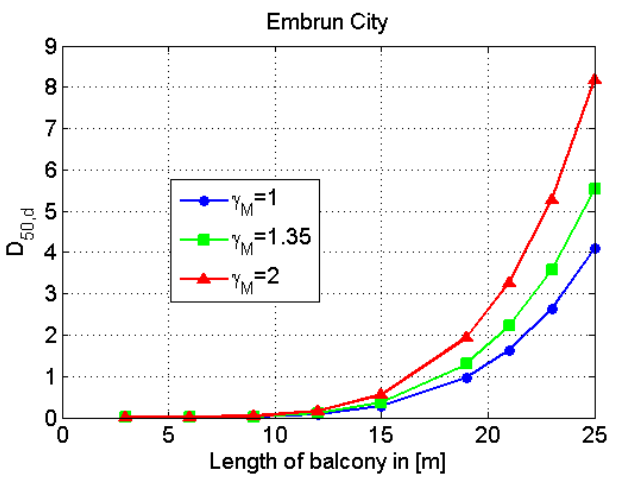

Fig. 19. Evolution of $\mathrm{D}_{50, \mathrm{~d}}$ in function of $\mathrm{L}_{\mathrm{b}}$ in Embrun with different values of $\gamma_{m}$

\section{Conclusions and perspectives}

The procedure for verifying the thermal break system SUNE against low-cycle fatigue loads induced by the deformations of the balcony due to the variations of the temperature has been presented in this paper. Primarily, eight cyclic experimental tests of the system are performed with several loading amplitudes. On one hand, 
they serve to define the fatigue design curve. On the other hand, they allow to develop a coupled plastic-damage mechanical model of the device. Then, the past temperature variations taken from ECA \& D database for five different cities are scaled in order to comply with the extreme values recommended in the Eurocodes. After adding the effect of the solar radiations, the elongation histories are computed for different lengths of balconies. Force-displacement histories are deduced using the mechanical model, and the cycles applied to the system are counted by a rainflow algorithm. Finally, the fatigue design curve allows to verify the fatigue strength of the thermal break for the different configurations considered.

As could be expected, the annual accumulated damage is larger in mountainous regions than in tempered areas, and the evolution of the damaging follows the same trends as the minimum and maximum shade air temperatures defined in the French national annex of the Eurocode.

The design format and the related safety coefficients are not known at this stage. But, it is clear that a safe design can be achieved for usual lengths of balconies, at least up to $12 \mathrm{~m}$. Moreover, a parametric study shows that the maximum length of the balcony is not proportional to the safety coefficient and that, even with a safety coefficient of 2 , a balcony length of $16 \mathrm{~m}$ can be reached in the most unfavorable location considered in the study.

This specific case illustrates that it is possible to exceed the conventional yielding limit during service life without any risk for the integrity of the components made with stainless steel. This offers a wide range of new possibilities, particularly in the case of shear keys used in thermal break systems.

Further investigations through reliability analysis are however needed in order to define an accurate safety format.

\section{Acknowledgements}

The authors gratefully acknowledge financial support by the ANR (Agence Nationale de la Recherche, France) through the project LabCom ANR B-HYBRID.

\section{References}

[1] Keo P, Le Gac B, Somja H, Palas F. Experimental Study of the Behavior of a SteelConcrete Hybrid Thermal Break System Under Vertical Actions. High Tech Concrete: Where Technology and Engineering Meet, Springer 2017; 2573-2580,

[2] AFNOR. NF A03-403-1990, Metal products : Low-cycle fatigue tests. Produits métalliques : Pratique des essais de fatigue oligocyclique, 1990/12/1

[3] Coffin Jr LF. A study of the effects of cyclic thermal stresses on a ductile metal, Trans. ASME 1954; 76:931-950.

[4] Manson SS. Behavior of materials under conditions of thermal stress, Tech. Rep., National Advisory Committee for Aeronautics, 1954.

[5] Bannantine J. Fundamentals of metal fatigue analysis, Prentice Hall, 1990.

[6] Jahed H, Varvani-Farahani A. Upper and lower fatigue life limits model using energy-based fatigue properties, International Journal of Fatigue 2006;28 (5) : 467-473.

[7] EN 1990, Eurocode 0: Basis of structural design, European Committee for Standardization, 2002.

[8] EN 1993-1-9, Eurocode 3: Design of steel structures: Part 1-9: Fatigue, European Committee for Standardization, 2005.

[9] Klein A, Tank J, Wijngaard G, Konnen G, Bohm R, Demaree J, Gocheva A, Mileta M, Pashiardis S, Hejkrlik L, Kern-Hansen C. Daily dataset of 20th-century surface air temperature and precipitation series for the European Climate Assessment. International Journal of Climatology 2002; 22 (12) : 1441-1453.

[10]EN 1991, Eurocode 1: Actions on structures: Part 1-5: General actions - Thermal actions, European Committee for Standardization, 2004.

[11]Nieslony A. Determination of fragments of multiaxial service loading strongly influencing the fatigue of machine components. Mechanical Systems and Signal Processing 2009; 23(8) : 2712-2721 